\title{
Perfectly absorbed and emitted currents by complex potentials in nonlinear media
}

\author{
Dmitry A. Zezyulin ${ }^{1}$ and Vladimir V. Konotop ${ }^{2}$ \\ ${ }^{1}$ ITMO University, St. Petersburg 197101, Russia \\ ${ }^{2}$ Departamento de Física and Centro de Física Teórica e Computacional, Faculdade de Ciências, \\ Universidade de Lisboa, Campo Grande 2, Edifício C8, Lisboa 1749-016, Portugal
}

(Dated: November 15, 2021)

\begin{abstract}
Recently it was demonstrated that the concept of a spectral singularity (SS) can be generalized to waves propagating in nonlinear media, like matter waves or electromagnetic waves in Kerr media. The corresponding solutions represent nonlinear currents sustained by a localized linear complex potential in a nonlinear Schrödinger equation. A key feature allowing the nonlinear generalization of a SS is a possibility to reduce a nonlinear current to the linear limit, where a SS has the unambiguous definition. In the meantime, known examples of nonlinear modes bifurcating from linear spectral singularities are few and belong to the specific class of constant-amplitude waves. Here we propose to extend the class of nonlinear SSs by incorporating solutions whose amplitudes are inhomogeneous. We show that the continuation from the linear limit requires a deformation of the complex potential, and this deformation is not unique. Examples include the deformation preserving the gain-and-loss distribution and the deformation preserving geometry of the potential. For the case example of a rectangular potential, we demonstrate that the nonlinear currents can be divided into two types: solutions of the first type bifurcate from the linear spectral singularities, and solutions of the second type cannot be reduced to the linear limit.
\end{abstract}

\section{INTRODUCTION}

Spatially localized complex potentials in the linear Schrödinger equation, subject to specific constraints on their characteristics, can result in total absorption [15. of the incoming waves or emission of outgoing waves. Physically, such regimes are referred to as coherent perfect absorption (CPA) [3, 6, 7] or lasing, respectively. Mathematically, the respective phenomena are related [6, 8, 9, to spectral singularities (SSs) emerging in the continuous spectra of the underlying complex potentials [10. The one-to-one correspondence between a solution corresponding to a spectral singularity (i.e., SSsolution) and a wave either incoming to or outgoing from the localized complex potential has been proven in [9] (see also [11]). Using this equivalence, in the one-dimensional geometry (which is assumed below), a linear SS-solution can be defined as a nontrivial solution $\phi_{0}(x)$ of a stationary Schrödinger equation with the asymptotic behavior $\phi_{0}(x) \rightarrow \rho_{ \pm} e^{i \varphi_{ \pm} \pm i k_{0} x}$ at $x \rightarrow \pm \infty$, where $\rho_{ \pm}$and $\varphi_{ \pm}$are real constants, and a nonzero real $k_{0}$ characterizes the location of the SS in the continuous spectrum. Positive and negative values of $k_{0}$ correspond to outgoing (laser) and incoming (CPA) wave boundary conditions, respectively. Therefore any SS corresponds to a peculiar scattering state in the continuous spectrum. In this respect, SSs are distinctively different from exceptional points (EPs) [12, 13, i.e., non-Hermitian degeneracies emerging from a coalescence of several square-integrable eigenfunctions from the discrete spectrum.

Considering electromagnetic waves in nonlinear Kerr media or superfluid flows in Bose-Einstein condensates (BECs), where two-body interactions define the properties of the background state, one has to take into account the nonlinearity of the respective waveguiding medium. The impact the nonlinearity is described by the nonlin- ear Schrödinger (NLS) equation (or the Gross-Pitaevskii equation in the case of BEC theory). Then a question about the extension of the concepts of CPA and laser to those nonlinear environments naturally arises. One can think of different implementations of the mentioned generalization. First, one can consider a nonlinear active layer that absorbs or emits waves, which is however embedded in a linear medium. In this case the effect of the nonlinearity will be confined to the localized spatial domain, but the emitted or absorbed waves remain linear, i.e., they still propagate in the linear medium [1418. Alternatively, one can consider a linear active potential operating in a nonlinear medium. In this case, the incoming or outgoing waves become nonlinear currents. Conceptually, such a possibility was elaborated in 19 21 and validated in experiments with atomic BECs [20] subjected to a localized dissipation. While there are obvious similarities between linear and nonlinear perfect absorption or lasing, one can point out four essential distinctions. First, while a linear monochromatic solution corresponding to a SS is characterized only by its frequency (wavelength or wavenumber) and by the relation between the right and left propagating waves at opposite infinities, in the nonlinear case the field amplitudes at the infinities must be equal, because now the frequency depends on the background amplitude (constancy of the frequency is only possible if the background amplitudes are equal at both infinities). Moreover, in the nonlinear system rescaling of the amplitude can have nontrivial impact on the absorption or lasing. Second, nonlinear currents can suffer from modulational instabilities (resulting from the nonlinearity of the medium rather from the presence of the gain or loss). Third, nonlinear currents cannot be explained by (only) the interference effects, which are known to be responsible for the coherent perfect absorption of linear waves [6]. Finally, the very concept 
of a SS is purely linear and cannot be uniquely defined in the nonlinear case, allowing multistable regimes [22]. Therefore, a key issue in the continuation of the linear perfectly absorbed or emitted currents to the nonlinear regime is the possibility to reduce the nonlinear system to a properly defined linear limit. Since the effective strength of nonlinearity is determined by the amplitude of the field, the linear limit can be associated with a regime where the field amplitude vanishes. Using this idea, several examples of CPAs and lasers for nonlinear waves have been elaborated. However, the known examples are still limited to few exceptional cases, including Dirac delta-potentials and Wadati potentials [19, 23, 24, where the linear solution is constant-amplitude and its generalization to the nonlinear case is obvious. In a more general situation, where the linear solution is not constant-amplitude, the nonlinear generalization is not that straightforward. The reason is that both nonlinear currents and linear SS-solutions require a delicate balance between the parameters of the potential and of the current itself. Any change of the wave parameters (e.g., the wavenumber) or rescaling of the amplitude (which becomes relevant in the nonlinear case) can violate this balance. As a result, for the persistence of nonlinear currents one should simultaneously deform the complex potential itself. Therefore, the transition between the linear and nonlinear regimes implies not only the increase of the field amplitude, but also a continuous deformation of the potential. A more detailed study of possible continuations of linear spectral singularities to the nonlinear medium is the first goal of the present article. We show that the deformation of the potential that enables the continuation to the nonlinear regime is not unique. Two examples that we will address in more detail correspond to the continuation that leaves the imaginary part of the potential intact and to the continuation that preserves the shape of the potential. In the latter case, the potential has rectangular shape either in linear and nonlinear cases, but the complex coefficient that governs the "strength" of the of the rectangular slab depends on the background field amplitude.

A distinctive feature of linear and nonlinear perfectly absorbed or emitted currents consists in specific boundary conditions which imply that sufficiently far from the potential the solution transforms into a purely incoming (for CPA) or purely outgoing (for laser) wave. In the meantime, some nonlinear perfectly absorbed currents that satisfy the boundary conditions of this type have already been considered in the literature, say in [25] 27, where these solutions have been interpreted in the context of the macroscopic Zeno effect, due to its resemblance to the well-known quantum Zeno effect 28 30. However, the eventual relation of these solutions to linear and nonlinear spectral singularities has not been discussed in any detail. A clarification of this issue is the second goal of the present article. Rather remarkably, we find that there exist nonlinear perfectly absorbed currents that cannot be reduced to the linear limit by chang- ing the coefficient of the rectangular potential. Therefore perfectly absorbed or emitted nonlinear currents can be classified in two types: solutions of the first type bifurcate from linear spectral singularities, and the nonlinear currents of the second type do not admit the described linear limit.

The plan of this article is as follows. The model and main conventions are introduced in Sec. III. In Sec. III we describe a continuation of a linear SS-solution to the nonlinear domain, considering the solution amplitude as a bifurcation parameter. The procedure developed in this section does not affect the gain-and-loss distribution of the complex potential. In Sec. [V] we propose a different approach to the nonlinear continuation which is illustrated for the case example of the rectangular potential. Section $\mathrm{V}$ compares the nonlinear currents bifurcating from the linear limit and the essentially nonlinear currents that cannot be reduced to the linear limit. The results are summarized in the concluding Sec. VI

\section{THE MODEL}

We consider a dimensionless field $\Phi(t, x)$ governed by the one-dimensional defocusing NLS equation

$$
i \Phi_{t}=-\Phi_{x x}+U(x) \Phi+|\Phi|^{2} \Phi,
$$

where $t$ and $x$ correspond to the evolution and transverse coordinates, respectively, and $U(x)$ is a localized complex potential:

$$
\lim _{x \rightarrow \pm \infty} U(x)=0
$$

that decays fast enough. While hereafter we use dimensionless units, it is known that in the BEC theory $\Phi(t, x)$ is a macroscopic wavefunction [31] and $t$ is time, while in the case of a beam propagating in a Kerr medium $\Phi(t, x)$ is a complex amplitude of the transverse electric field and $t$ is a coordinate along the propagation axis 32. In the BEC context, the complex potential $U(x)$ can be created either by elimination and loading BEC atoms, while for the optical beam the potential is determined by the complex dielectric permittivity of the cubic medium.

We consider stationary solutions $\Phi(t, x)=e^{-i \mu t} \phi(x)$, where $\mu$ is a real parameter (depending on the physical context, it is a dimensionless chemical potential of the condensate or describes the change of the propagation constant of the optical beam). The stationary wavefunction $\phi$ satisfies

$$
-\phi_{x x}+U(x) \phi+|\phi|^{2} \phi=\mu \phi .
$$

We are interested in solutions of (3) subject to the finite density boundary conditions, i.e., when $|\phi(x)|$ tends to a nonzero constant at the infinity. In view of (2), the only boundary condition of this type consistent with (3) is $|\phi(x)| \rightarrow \rho>0$ at $x \rightarrow \pm \infty$. Respectively, we say that $\phi(x)$ is a perfectly absorbed (emitted) current with 


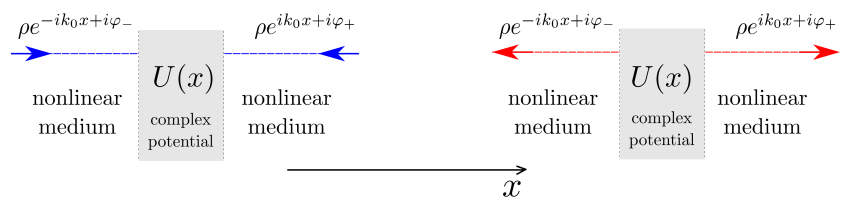

FIG. 1. Schematic illustration boundary conditions for the $\mathrm{CPA}$ at $k_{0}<0$ (left panel) and laser at $k_{0}>0$ (right panel).

background amplitude $\rho$ if there exist real phases $\varphi_{ \pm}$and real constant $k_{0}<0\left(k_{0}>0\right)$ such that (see schematics in Fig. 1)

$$
\lim _{x \rightarrow-\infty} \phi(x) e^{i k_{0} x}=\rho e^{i \varphi_{-}}, \quad \lim _{x \rightarrow \infty} \phi(x) e^{-i k_{0} x}=\rho e^{i \varphi_{+}} .
$$

For such solutions $\mu=\rho^{2}+k_{0}^{2}$.

Generally, a solution of (1), (4) with fixed amplitude $\rho$ and phases $\varphi_{ \pm}$may exist only for specific choices of the complex potential $U(x)$ which depends on the background amplitude $\rho$. Therefore below we denote such solutions and potentials by $\phi(x)=\phi_{\rho}(x)$ and $U(x)=$ $U_{\rho}(x)$, respectively, where subscript $\rho$ emphasizes the dependence on background amplitude. Suppose now that there exist the limits

$$
\lim _{\rho \rightarrow 0} \frac{\phi_{\rho}(x)}{\rho}=: \phi_{0}(x), \quad \lim _{\rho \rightarrow 0} U_{\rho}(x)=: U_{0}(x) .
$$

Then $\phi_{0}(x)$ is a SS-solution of the linear problem

$$
\begin{aligned}
& {\left[-\partial_{x}^{2}+U_{0}(x)\right] \phi_{0}=k_{0}^{2} \phi_{0},} \\
& \lim _{x \rightarrow \pm \infty} \phi_{0}(x) e^{\mp i k_{0} x}=e^{i \varphi_{ \pm}}
\end{aligned}
$$

which will be considered as the definition of the linear limit of the problem (1), (4).

Considering the linear limit alone, i.e., not related to the nonlinear problem (1), (4), the boundary conditions for (7) can be generalized to unequal amplitudes $\rho_{ \pm}>0$ at the infinities

$$
\lim _{x \rightarrow \pm \infty} \phi_{0}(x) e^{\mp i k_{0} x}=\rho_{ \pm} e^{i \varphi_{ \pm}} .
$$

Since in the linear problem the field amplitude can be scaled out, although the constants $\rho_{+}$and $\rho_{-}$are allowed to be different, only the relation between them, $\rho_{+} / \rho_{-}$, is a relevant parameter. Thus, given a linear SS solution $\phi_{0}(x)$, it can be a limit of the nonlinear absorbing or lasing current only if $\rho_{+} / \rho_{-}=1$. Notice, however, that we are considering linear and nonlinear currents corresponding to the same $k_{0}$ (i.e., to the same wavelength).

From (3) and (4) it is straightforward to obtain a useful relation

$$
2 k_{0} \rho^{2}=\operatorname{Im} \int_{-\infty}^{\infty} U_{\rho}(x)\left|\phi_{\rho}\right|^{2} d x .
$$

It provides a mathematical expression of the obvious fact that perfectly absorbed or emitted currents cannot be obtained in conservative systems $\left[\operatorname{Im} U_{\rho}(x) \neq 0\right.$ is a necessary conditions for existence of such solutions]. More generally, it follows from $(9)$ that $\mathcal{P} \mathcal{T}$-symmetric potentials, for which $U_{\rho}(x)=\mathcal{P} \mathcal{T} U_{\rho}(x)=U_{\rho}^{*}(-x)$, do not support $\mathcal{P} \mathcal{T}$-symmetric currents characterized by $\left|\phi_{\rho}(x)\right|^{2}=\left|\phi_{\rho}(-x)\right|^{2}$. From the physical point of view, Eq. (9) can be considered as a balance equation which relates the energy dissipated by the localized absorbing potential (or emitted by the localized gain) and the strength of the energy fluxes incoming from the infinity (or outgoing towards the infinity).

\section{CONTINUATION PRESERVING THE GAIN-AND-LOSS DISTRIBUTION}

It is known 23 that under certain (and not very restrictive) conditions, a linear SS-solution $\phi_{0}(x)$ exists if and only if the potential $U_{0}(x)$ in Eq. (6) admits the representation

$$
U_{0}(x)=-w^{2}(x)-i \frac{d w(x)}{d x}+k_{0}^{2},
$$

where $w(x)=w_{r e}(x)+i w_{i m}(x)$, with $w_{r e}(x)=\operatorname{Re} w(x)$ and $w_{i m}(x)=\operatorname{Im} w(x)$, is a complex-valued base function with asymptotic behavior

$$
\lim _{x \rightarrow \pm \infty} w(x)=\mp k_{0} .
$$

If additionally $w(x)$ is a continuous function of $x$, then the linear solution allows for a universal representation through the base function

$$
\phi_{0}(x)=c_{0} \exp \left[-i \int_{x_{0}}^{x} w(\xi) d \xi\right],
$$

where $x_{0}$ is an arbitrary point of the real axis, and constant $c_{0}$ is introduced to ensure the boundary conditions (7):

$$
c_{0}=\exp \left[\int_{-\infty}^{x_{0}} w_{i m}(\xi) d \xi\right] .
$$

With some technical amendments, the consideration can be generalized on the case when $w(x)$ has discontinuities of certain form 23 .

The universal form (12) can be used for construction of nonlinear currents bifurcating from the linear limit. Indeed, first we notice that (7) imposes the following constraint on the base function

$$
\int_{-\infty}^{\infty} w_{i m}(x) d x=0
$$

Next, we observe that solution $\phi_{\rho}(x)=\rho \phi_{0}(x)$, where $\phi_{0}(x)$ is defined by $(12)$, satisfies the nonlinear equation (3) with the deformed potential

$$
U_{\rho}(x)=U_{0}(x)+\rho^{2}\left(1-\exp \left[-2 \int_{x}^{\infty} w_{i m}(\xi) d \xi\right]\right) .
$$


Thus, constraint (14) can be viewed as a sufficient condition for a possibility of continuation of a linear SSsolution without zeros on the real axis [i.e., the one allowing the representation (12)] into the nonlinear domain by a continuous deformation of the potential (15), where $\rho$ is a bifurcation parameter. Condition (14) is rather general: say, it holds if the potential $U_{0}(x)$ is an even function. Deformation (15) only affects the real part of the potential, while the imaginary part of $U_{\rho}(x)$ does not depend on $\rho$. In a special case when $w(x)$ is purely real, i.e., $w_{i m}(x) \equiv 0$, no deformation of the potential is necessary at all. This reflects the fact that the respective solution $\phi_{\rho}(x)=\rho \phi_{0}(x)$ has a constant amplitude, i.e., $|\phi(x)| \equiv \rho$.

As we mentioned above, if

$$
\int_{-\infty}^{\infty} w_{i m}(x) d x=\gamma \neq 0
$$

then no weak deformation of the respective linear potential $U_{0}(x)$ admits continuation of the linear SS-solution to nonlinear regime. In the meantime, allowing spatially unconfined deformations one can define a meaningful smallamplitude limit of the respective nonlinear currents. Indeed, let $f(x)$ be an arbitrary sufficiently well-behaved real-valued function such that

$$
\int_{-\infty}^{\infty} f(x) d x=\gamma
$$

Define

$$
\hat{w}(x)=w(x)-i \rho^{2} f\left(\rho^{2} x\right) .
$$

Obviously $\hat{w}(x)$ satisfies 14 , i.e.,

$$
\operatorname{Im} \int_{-\infty}^{\infty} \hat{w}(x) d x=0
$$

and has the required asymptotic behavior (11). We therefore can use this function to construct a nonlinear SSsolution using expressions 12 - 13 , where $w(x)$ is to be replaced with $\hat{w}(x)$. Respectively, the deformation of the potential is given as

$$
\begin{aligned}
U_{\rho}(x)= & -\hat{w}^{2}-i \hat{w}_{x}+k_{0}^{2} \\
& +\rho^{2}\left(1-\exp \left[-2 \operatorname{Im} \int_{x}^{\infty} \hat{w}(\xi) d \xi\right]\right)
\end{aligned}
$$

Notice however that the deformation of the potential is nonlocal in the sense that the region of its localization, that can be estimated as $|x| \lesssim 1 / \rho$, tends to infinity as $\rho$ approaches zero.

\section{CONTINUATION PRESERVING THE SHAPE OF THE POTENTIAL: CASE STUDY FOR THE RECTANGULAR BARRIER}

We have demonstrated in the previous section that any linear SS-solution with equal background amplitudes at the infinities can be continued to the nonlinear regime with a proper deformation of only real part of the linear potential [see (15)]. Since deformations of the imaginary part of a linear potential can also be considered, now, using a particular example of a rectangular potential, we demonstrate that continuation of the SS-solutions to the nonlinear domain is not unique. To this end, we define the rectangular function

$$
\operatorname{rect}(x):=\left\{\begin{array}{l}
1 \text { if }|x| \leq 1 \\
0 \text { if }|x| \geq 1
\end{array}\right.
$$

and consider the potential

$$
U_{\rho}(x)=\zeta_{\rho} \operatorname{rect}(x),
$$

where $\zeta_{\rho}$ is a complex coefficient. As before, we use subscript $\rho$ to parameterize the deformation of the potential for different values of the background amplitude, and $\rho=0$ corresponds to the linear limit. Hence the potential 22 remains rectangular upon the change of $\zeta_{\rho}$.

\section{A. Linear spectral singularities}

The linear SS-solutions of (6) with the rectangular potential have already been addressed in literature (see e.g. [1, 3, 4, 16, 23, 33 35]). For completeness, herein we recall some of the known results. Consider the linear problem (6) with rectangular potential $U_{0}(x)$ defined by (22) with $\zeta_{\rho}=\zeta_{0}$. In order to identify the corresponding SS-solutions, it is convenient to use the representation $\zeta_{0}=k_{0}^{2}-\kappa_{0}^{2}$, where $\kappa_{0}$ is a new complex parameter. Then, under the condition

$$
k_{0}=i \kappa_{0} \tan \kappa_{0},
$$

there exists an even SS-solution

$$
\phi_{0}^{\text {even }}(x)= \begin{cases}e^{i k_{0}(|x|-1)} & \text { if }|x| \geq 1 \\ \cos \left(\kappa_{0} x\right) / \cos \kappa_{0} & \text { if }|x| \leq 1\end{cases}
$$

This SS-solution and the corresponding potential $U_{0}(x)$ can be represented in the form $\sqrt{12}$ and 10 with the base function defined as 23]

$$
w(x)= \begin{cases}\mp k_{0} & \text { if } \pm x \geq 1 \\ -i \kappa_{0} \tan \left(\kappa_{0} x\right) & \text { if }|x| \leq 1\end{cases}
$$

On the other hand, under the condition

$$
k_{0}=-i \kappa_{0} \cot \kappa_{0}
$$

there is an odd SS-solution

$$
\phi_{0}^{\text {odd }}(x)= \begin{cases} \pm e^{i k_{0}(|x|-1)} & \text { if } \pm x>1 \\ \sin \left(\kappa_{0} x\right) / \sin \kappa_{0} & \text { if }|x| \leq 1\end{cases}
$$



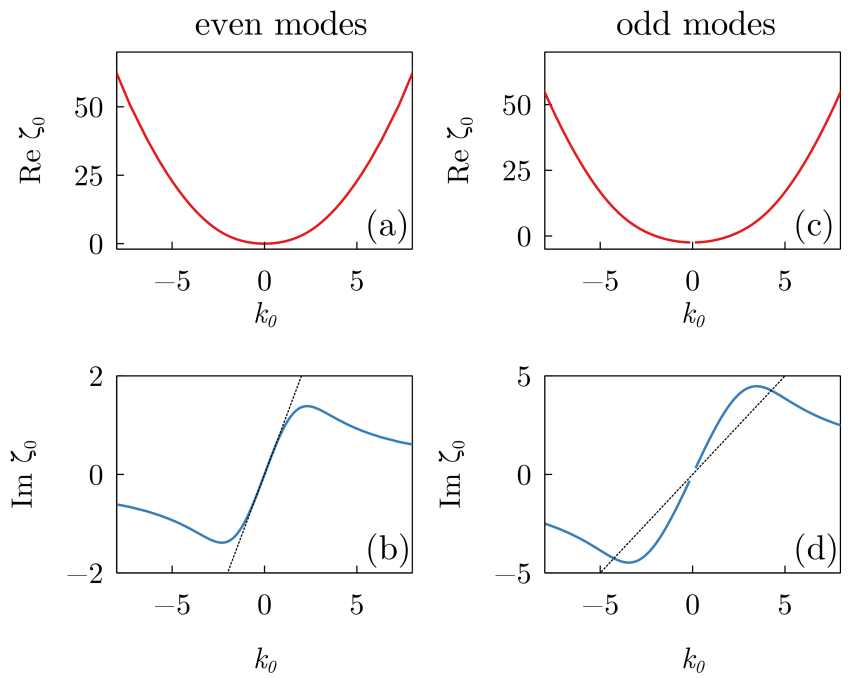

FIG. 2. Complex coefficient $\zeta_{0}$ of rectangular potential corresponding to spectral singularities in the linear limit for even $(\mathrm{a}, \mathrm{b})$ and odd $(\mathrm{c}, \mathrm{d})$ modes. Positive and negative $k_{0}$ correspond to lasing and absorption, respectively. Dotted lines in (b,d) correspond to $\operatorname{Im} \zeta_{0}=k_{0}$.

For the odd solution the base function in 12 and 100 has the form

$$
w(x)= \begin{cases}\mp k_{0} & \text { if } \pm x \geq 1 \\ i \kappa_{0} \cot \left(\kappa_{0} x\right) & \text { if }|x| \leq 1\end{cases}
$$

Solving Eqs. (23) and (26), we find that for each $k_{0} \neq 0$ there exist one even and one odd linear mode. Dependencies of the complex parameter $\zeta_{0}$ on $k_{0}$ for linear spectral singularities are shown in Fig. 2, We notice that for even modes the inequality $\left|\operatorname{Im} \zeta_{0}\right|<\left|k_{0}\right|$ is valid. For odd modes the latter inequality holds only for wavenumbers of sufficiently large modulus, whereas for small $k_{0}$ the opposite inequality takes place, i.e., $\left|\operatorname{Im} \zeta_{0}\right|>\left|k_{0}\right|$.

\section{B. Bifurcations from linear SS-solutions}

Let $\phi_{0}(x)$ be the linear SS-solution defined either by Eq. (24) or by Eq. (27). Apart from $\phi_{0}$, linear equation (6) has a linearly independent solution $\tilde{\phi}_{0}(x)$ which can be written as

$$
\tilde{\phi}_{0}(x)=\phi_{0}(x) \int_{0}^{x} \phi_{0}^{-2}(\xi) d \xi .
$$

The solutions $\phi_{0}(x)$ and $\tilde{\phi}_{0}(x)$ are of opposite parities and their Wronskian is identically equal to unity: $\phi_{0} \tilde{\phi}_{0, x}-$ $\phi_{0, x} \tilde{\phi}_{0} \equiv 1$.

In order to construct a continuation of the SS-solution $\phi_{0}$ for the nonlinear equation (3), we treat $\rho$ as a small parameter and introduce the following asymptotic expansions for the solution and for the coefficient of the rect- angular potential:

$$
\begin{array}{r}
\phi_{\rho}(x)=\rho \phi_{0}(x)+\sum_{m=1}^{\infty} \rho^{2 m+1} q_{m}(x), \\
\zeta_{\rho}=\zeta_{0}+\sum_{m=1}^{\infty} \rho^{2 m} z_{m},
\end{array}
$$

where $q_{m}(x)$ and $z_{m}$ are coefficients to be found. Formal existence of asymptotic expansions (30) for all orders of $m$ can be established in the straightforward way. Indeed, substituting (30) into the nonlinear equation (3) and collecting the terms with equal powers of $\rho$, we observe that in $\rho$ - and $\rho^{2}$-orders the resulting equations are satisfied automatically. The first nontrivial equation arises at $\rho^{3}$ order and reads

$$
\left[\partial_{x}^{2}+k_{0}^{2}-U_{0}(x)\right] q_{1}(x)+f_{1}(x)=0,
$$

where

$$
f_{1}(x)=\left[1-\left|\phi_{0}(x)\right|^{2}-z_{1} \operatorname{rect}(x)\right] \phi_{0}(x) .
$$

A general solution of Eq. 311 can be written in the form

$$
\begin{aligned}
q_{1}(x)=C_{1} \phi_{0}(x)+C_{2} \tilde{\phi}_{0}(x) & -\tilde{\phi}_{0}(x) \int_{0}^{x} \phi_{0}(\xi) f_{1}(\xi) d \xi \\
& +\phi_{0}(x) \int_{0}^{x} \tilde{\phi}_{0}(\xi) f_{1}(\xi) d \xi,
\end{aligned}
$$

where $C_{1,2}$ are, so far, arbitrary constants. Looking for a solution that has the same parity as $\phi_{0}(x)$ does, we set $C_{2}=0$. Since the function $f_{1}(x)$ is identically zero outside the interval $[-1,1]$, we can define the integrals

$$
\begin{aligned}
& \lambda_{1}=\int_{0}^{\infty} \phi_{0}(\xi) f_{1}(\xi) d \xi=\int_{0}^{1} \phi_{0}(\xi) f_{1}(\xi) d \xi \\
& \tilde{\lambda}_{1}=\int_{0}^{\infty} \tilde{\phi}_{0}(\xi) f_{1}(\xi) d x=\int_{0}^{1} \tilde{\phi}_{0}(\xi) f_{1}(\xi) d x
\end{aligned}
$$

Looking for a localized function $q_{1}(x)$, we require $\lambda_{1}=0$ and set $C_{1}=-\tilde{\lambda}_{1}$. The requirement $\lambda_{1}=0$ determines the first correction to the rectangular potential:

$$
z_{1}=\frac{\int_{0}^{1}\left(1-\left|\phi_{0}\right|^{2}\right) \phi_{0}^{2} d x}{\int_{0}^{1} \phi_{0}^{2} d x} .
$$

This quotient is generically complex, i.e., both real and imaginary part of the rectangular potential should be adjusted.

A similar procedure can be carried out for all higher powers of $\rho$ which determine further corrections to the complex coefficient of the potential. The correction $q_{1}(x)$ constructed in this way, as well as all higher-order corrections $q_{m}$ are identically zero outside the interval $[-1,1]$. Therefore a nonlinear perfectly absorbing or lasing solution constructed through this procedure coincide with the properly scaled linear SS-solution outside the support of the potential, i.e., $\phi_{\rho}(x)-\rho \phi_{0}(x) \equiv 0$ as $|x| \geq 1$. This in particular means that $\left|\phi_{\rho}(x)\right| \equiv \rho$ as $|x| \geq 1$. 

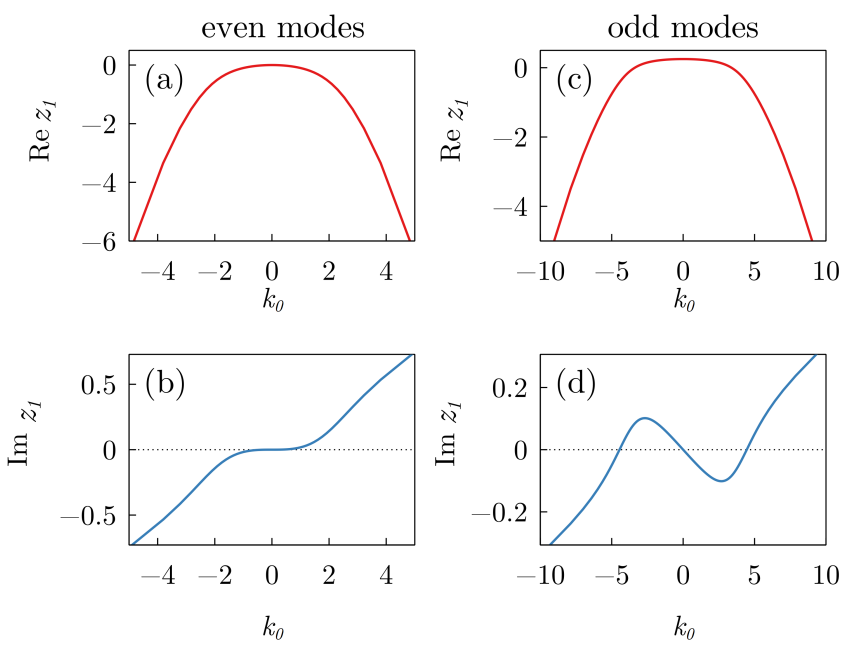

FIG. 3. Real and imaginary parts of the leading correction $z_{1}$ to the complex coefficient of the rectangular potential that features a SS in the linear limit vs. $k_{0}$.

\section{Numerical study of nonlinear currents}

Using (35), we computed the leading correction $z_{1}$ to the coefficient of the rectangular potential. The result is presented in Fig. 3 as a function of $k_{0}$. For even modes, illustrated in Fig. 3(a,b), the imaginary part of $z_{1}$ has the same sign as $k_{0}$. Since positive and negative imaginary part of the complex coefficient $\zeta_{\rho}$ correspond, respectively, to gain and loss, this means that lasing of weakly nonlinear even modes requires stronger gain, than its purely linear counterpart does, while absorption of nonlinear even modes requires stronger losses than those required by the linear CPA.

For odd modes illustrated in Fig. 3(c,d), the behavior of the leading correction $z_{1}$ is different. For small wavenumbers $k_{0}$ the imaginary part of the coefficient $z_{1}$ is negative for emitting modes and is positive for absorbing modes. In this interval of wavenumbers, the perfect absorption and lasing of nonlinear modes require, respectively, weaker losses and gain than those of the linear SS-solutions. However, for larger $\left|k_{0}\right|$ the sign of the imaginary part of $z_{1}$ coincides with the sign of $k_{0}$.

To validate the predictions of the asymptotic expansions (30), we have computed numerically several branches of perfectly absorbed nonlinear modes bifurcating from the linear limit. Typical results for even and odd currents are shown in Fig. 4(a-c) and Fig. 4(df), respectively. In the vicinity of the bifurcations from the linear limit at $\rho=0$, the real and imaginary parts of the complex coefficient $\zeta_{\rho}$ are well approximated by the analytical prediction (dashed lines). To describe the nonlinearity-induced deviation between the shapes of linear and nonlinear currents, for each amplitude $\rho$ we compute the integral measure

$$
I_{n l}=\int_{0}^{1}\left(\left|\phi_{\rho}\right|^{2}-\rho^{2}\left|\phi_{0}\right|^{2}\right) d x
$$
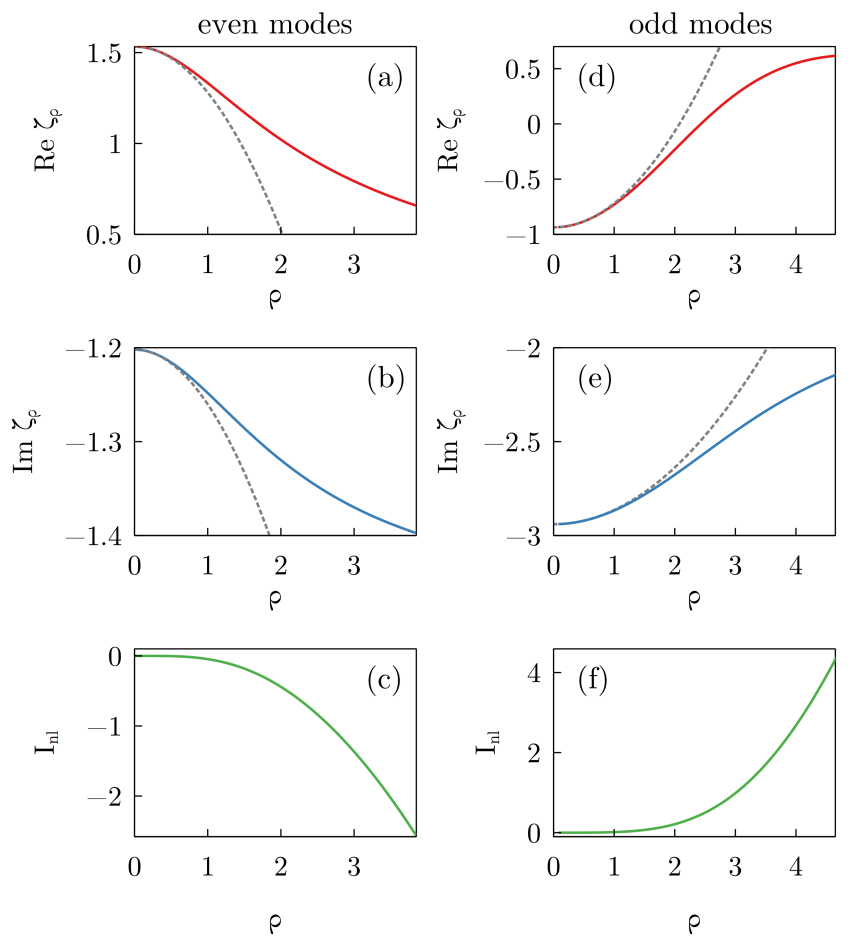

FIG. 4. Branches of nonlinear perfectly absorbing currents bifurcating from the linear limit which corresponds to zero background amplitude $\rho=0$. Upper and middle rows show real and imaginary parts of the complex coefficient $\zeta_{\rho}$ of the rectangular potential. Grey dashed lines plot asymptotic predictions $\zeta_{\rho}=\zeta_{0}+z_{1} \rho^{2}$ where $z_{1}$ is computed from (35). Lower panels (c) and (f) show the dependence of $I_{n l}$ on $\rho$. Even and odd modes correspond, respectively, to $k_{0} \approx-1.474$ and $k_{0} \approx-1.589$.
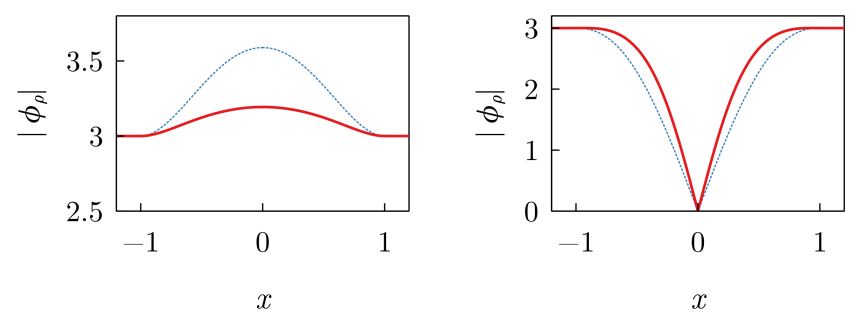

FIG. 5. Moduli $\left|\phi_{\rho}(x)\right|$ of even and odd nonlinear perfectly absorbed currents (red curves) selected from Fig. 4 at $\rho=$ 3 juxtaposed to moduli of their linear counterparts $\rho\left|\phi_{0}(x)\right|$ which are normalized to have the same background amplitude (blue dashed lines).

This quantity, plotted in the lower panels of Fig. 4 is negative for even modes and positive for odd modes. Figure 5 compares the shapes of nonlinear SS solutions with their linear counterparts.

While in the linear context CPA and lasing can be considered as equivalent modulo to time-reversal [6], the corresponding nonlinear regimes are not equivalent from the point of view of stability. Here we examine the linear stability of perfectly absorbed absorbed currents using 
the standard analysis [36]. A numerical study of linear stability spectra indicates that even modes from Fig. 4 are stable both in the lasing and absorbing cases, whereas odd modes are stable only in the absorbing regime.

\section{ESSENTIALLY NONLINEAR PERFECTLY ABSORBED CURRENTS}

A characteristic property of currents constructed in the previous section is that the field amplitude of solutions (both linear and nonlinear) is exactly constant outside the support of the rectangular potential, i.e., $\left|\phi_{\rho}(x)\right| \equiv \rho$ if $|x| \geq 1$. Apart from these solutions, the nonlinear medium supports a different type of perfectly absorbed currents for which the background density is approached asymptotically, i.e., $\lim _{x \rightarrow \pm \infty}\left|\phi_{\rho}(x)\right|=\rho$. While currents of this type have been introduced in [25] for a purely imaginary localized potential, their eventual relation to linear SSs and the behavior near to the linear limit have not been addressed yet. To clarify these issues, here we numerically investigate this type of nonlinear perfectly absorbed currents considering the rectangular potential $(22)$. We will demonstrate that the perfectly absorbed solutions with the asymptotically approached background amplitude cannot be reduced to the linear SS-solutions. Therefore, the nonlinear perfectly absorbed currents in the rectangular potential can be classified in two different types: solutions of the first type [described in the previous section [V] bifurcate from the spectral singularities in the linear limit, while solutions of the second type [considered in the present section] do not admit the linear limit.

To compute nonlinear currents of the second type, we again use the nonlinear equation (3) with boundary conditions (4). In comparison with nonlinear currents of the first type, the perfectly absorbed currents of the second type are easier-to-find, because perfect absorption at given $k_{0}$ and background amplitude $\rho$ can be achieved by tuning only real or only imaginary part of the coefficient $\zeta_{\rho}$. In Fig. 6 we present diagrams $\left(\operatorname{Re} \zeta_{\rho}, \rho\right)$ for the rectangular potentials enabling perfectly absorbed currents at a given $k_{0}$ and fixed $\operatorname{Im} \zeta_{\rho}$. The dependencies feature peculiar snaking behavior, for both even and odd currents, and, most importantly, neither dependency can be continued to the linear limit $\rho \rightarrow 0$. Non-existence of the linear limit for nonlinear currents of this type agrees with the inequality

$$
\rho>\sqrt{2}\left|k_{0}\right|
$$

which was obtained in 25] from the analysis of the asymptotic behavior of the spatial profiles of the currents for $x \rightarrow \pm \infty$. Spatial profiles of perfectly absorbed states of the second type (exemplified in Fig. 7) are distinctively different from the currents of the first type found in Sec. IV] Multiple local minima and maxima of the amplitude for solutions in Fig. 7(B,D) can
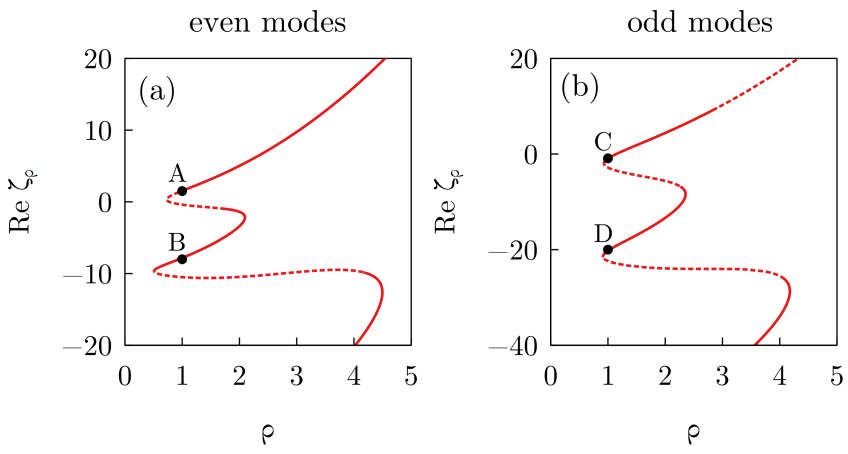

FIG. 6. Dependencies of $\operatorname{Re} \zeta_{\rho}$ on $\rho$ for even and odd nonlinear perfectly absorbed currents without linear counterpart, while $k_{0}$ and $\operatorname{Im} \zeta_{\rho}$ remain fixed. For even modes $k_{0} \approx-0.217$ and $\operatorname{Im} \zeta_{\rho} \approx-1.202$. For odd modes $k_{0} \approx-0.291$ and $\operatorname{Im} \zeta_{\rho} \approx-2.940$. Solid and dotted fragments correspond to stable and unstable solutions, respectively. Points labeled AD correspond to the specific solutions plotted in Fig. 7(A-D).

be understood by noticing that real parts of the corresponding rectangular potentials are negative with large absolute values (see points B and D in Fig. 6). In this situation the oscillating shape of the solution inside the rectangular slab can be approximated by the Jacobian elliptic sine: $\phi \approx \sqrt{2 m} \nu \operatorname{sn}\left(\nu\left(x-x_{0}\right), m\right)$, where $m$ is the so-called parameter [37] and the real coefficient $\nu$ characterizes the frequency of spatial oscillations. For a strong rectangular potential one can roughly estimate $\nu \approx \sqrt{\left|\operatorname{Re} \zeta_{\rho}\right| /(m+1)}$, which corresponds to fast spatial oscillations. Qualitatively such a spatial dependence of nonlinear currents resembles that of the linear SSsolutions: see Eqs. (24) and (27), where for $\zeta_{0}$ with large and negative real part we estimate $\kappa_{0} \approx \sqrt{\left|\operatorname{Re} \zeta_{0}\right|}$ (i.e., the same energy in a more deep potential corresponds to smaller wavelength of the eigenmode).

The linear stability study for nonlinear currents of the second type reveals several alternating regions with stable (solid lines in Fig. 6) and unstable (dashed lines in Fig. 6) currents.

So far, our consideration has been focused on deformation of a complex potential for the wavenumber $k_{0}$ being fixed both in linear and in nonlinear regimes. Obviously, an alternative statement is also meaningful: one can fix the imaginary potential and consider the change of $k_{0}$ under the variation of the background amplitude $\rho$. To address this possibility, we fix real and imaginary parts of the amplitude of the rectangular potential, denoting it by $\zeta$, and consider the small-amplitude limit $\rho \rightarrow 0$ allowing the wavenumber $k_{0}$ to change. The corresponding diagrams are presented in Fig. 8, where we observe that in the small-amplitude limit the wavenumber vanishes, $\lim _{\rho \rightarrow 0} k_{0}=0$, i.e., for small $\rho$ the absorption is effectively inhibited. Looking to the spatial shapes of the currents with small background amplitude $\rho$, we notice that the attenuation of the absorption is explained by the fact that the solution leaves the domain where the absorbing 

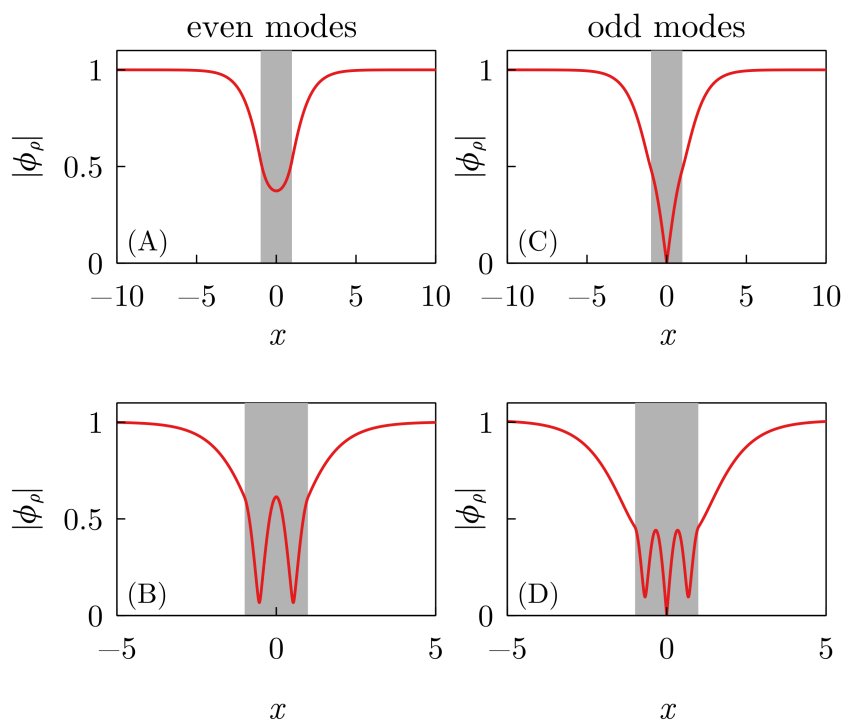

FIG. 7. Spatial profiles $\left|\phi_{\rho}(x)\right|$ for stable perfectly absorbed currents with $\rho=1$ from Fig. 6] Gray regions correspond to the domain $x \in[-1,1]$ there the absorbing potential is confined. Panels A-D correspond to the points A-D in Fig. 6.

potential is confined [Fig. 8, second row]. In the limit of large $\rho$ the amplitude of even states becomes almost constant $\left|\phi_{\rho}(x)\right| \approx \rho$ [Fig. $\left.8(\mathrm{c})\right]$. As readily follows from Eq. (9), for these solutions $\left|k_{0}\right|$ approaches $|\operatorname{Im} \zeta|$ from below as $\rho \rightarrow \infty$. The oscillatory behavior of the odd solution in Fig. 8(f) can be again explained in terms of Jacobian functions, because for $\rho \gg 1$ the wavefunction inside the slab can be approximated as $\phi \approx \sqrt{2 m} \nu \operatorname{sn}(\nu x, m)$, where $\nu \approx \sqrt{\rho /(m+1)}$.

Since for all currents of the second type the inequality $\left|\phi_{\rho}(x)\right| \leq \rho$ holds, using (9) we obtain that

$$
\left|k_{0}\right| \leq\left|\operatorname{Im} \zeta_{\rho}\right| \text {. }
$$

Let us now recall that for linear SSs we have observed that for even modes the inequality $\left|\operatorname{Im} \zeta_{0}\right|<\left|k_{0}\right|$ holds (see Fig. 2 and the discussion around). This leads us to the following conclusion for even currents: if the rectangular potential is chosen such that linear spectral singularity at $k_{0}$ coexist with nonlinear current of the second type at the same wavenumber $k_{0}$, then the nonlinear current of the second type requires stronger absorption than the linear current. Note that odd currents the situation can be more complex, because in this case the inequality $\left|\operatorname{Im} \zeta_{0}\right|<\left|k_{0}\right|$ holds only for sufficiently large $k_{0}$.

\section{CONCLUSION}

One-dimensional nonlinear Schrödinger equation with a localized complex potential and spatially-uniform Kerrtype cubic nonlinearity can support perfectly absorbed or emitted currents. Solutions of this class are characterized by purely incoming or purely outgoing wave boundary
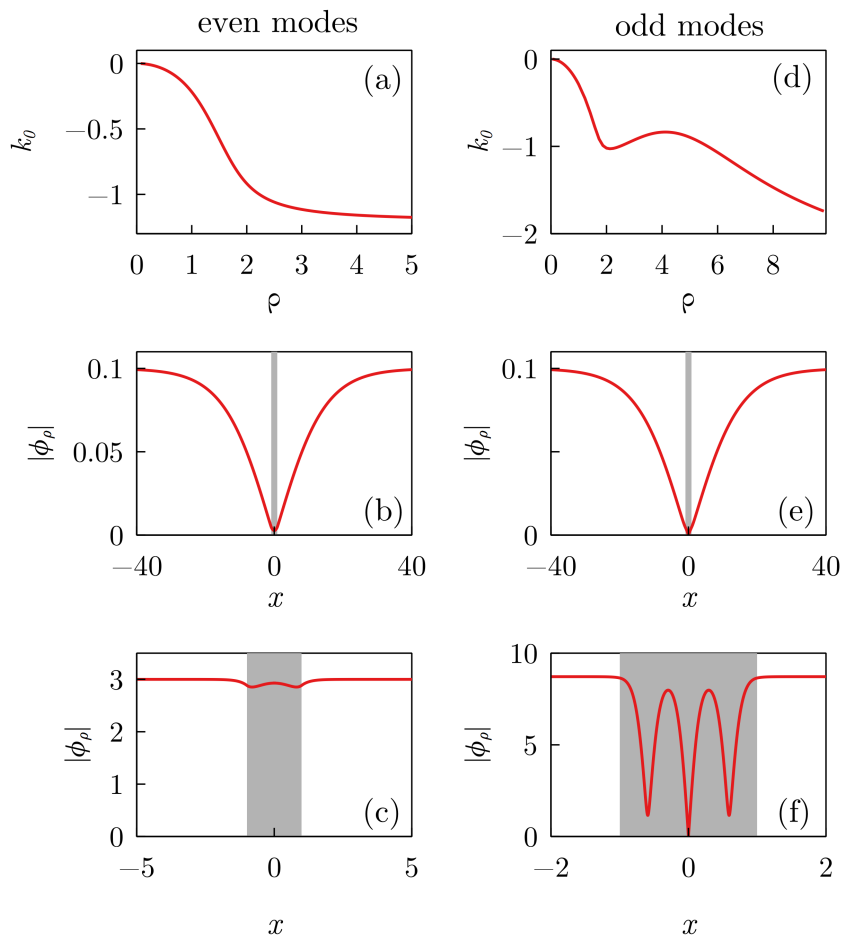

FIG. 8. (a,d) Dependencies of the wavenumber $k_{0}$ on the background amplitude $\rho$ for even and odd perfectly absorbed currents of the second type, while the complex coefficient $\zeta_{\rho}$ of the absorbing potential is kept fixed. Panels in two lower rows show spatial profiles $\left|\phi_{\rho}(x)\right|$ for even and odd modes with different background amplitudes. Gray regions correspond to the domain $x \in[-1,1]$ there the absorbing potential is confined. Shown solutions are stable. For even and odd currents, $\zeta \approx 1.533-1.202 i$ and $\zeta \approx-0.936-2.940 i$, respectively.

conditions and in this respect resemble spectral singularities which are well-known in the linear theory of nonHermitian operators. Therefore a question emerges on the possibility to transform from a nonlinear current to a spectral singularity (or back) by decreasing (or increasing) the effective nonlinearity strength. This question has been previously answered only for a very specific situation where the amplitude of the solution is constant. In the present paper, we have generalized the earlier treatment to non-constant-amplitude solutions. We have demonstrated that in the general situation bifurcation of a nonlinear current from a spectral singularity is possible only if the complex potential is deformed properly, and this deformation is not unique. For a case example of rectangular potential, bifurcations from the linear limit are possible only if the complex coefficient of the slab is tuned properly. In contrast to the linear case, nonlinear absorbing currents cannot be considered as antilasers, because perfectly absorbing and emitted currents feature different stability properties. Additionally, the rectangular potential supports essentially nonlinear currents that cannot be reduced to the linear limit. Solutions of this type are very different from those bifurcating from linear 


\begin{tabular}{ll} 
property & currents of \\
\hline linear limit & spectral sing \\
$\begin{array}{l}\text { field amplitude outside the potential } \\
\text { with a finite support }\end{array}$ & constant \\
$\begin{array}{l}\text { existence conditions at given wavenum- } \\
\text { ber and background amplitude }\end{array}$ & both real and \\
potential mu
\end{tabular}

\section{ACKNOWLEDGMENTS}

Work of D.A.Z. is funded by Russian Foundation for Basic Research (RFBR) according to the research project gular potential. currents of the second type

does not exist

space-dependent

it is sufficient to adjust only a real or only an imaginary part of the potential

$k_{0}^{2}<\rho^{2} / 2$

TABLE I. Summary of dissimilarities between the two types of nonlinear perfectly absorbed currents supported by the rectan-

spectral singularities. The main dissimilarities between the rectangular potential are summarized in Table 1 .

No. 19-02-00193. V.V.K. acknowledges financial support from the Portuguese Foundation for Science and Technology (FCT) under Contract UIDB/00618/2020.
[1] A. P. Khapalyuk, Dokl. Akad. Nauk BelSSR 6, 301 (1962).

[2] A. P. Khapalyuk, Opt. Spectrosk. 52, 194 (1982).

[3] N. N. Rosanov, Antilaser: Resonance absorption mode or coherent perfect absorption? Phys. Usp. 60, 818 (2017).

[4] A. A. Zharov and T. M. Zaboronkova, Fiz. Plazmy 9, 995 (1983).

[5] L. Poladian, Resonance mode expansions and exact solutions for nonuniform gratings, Phys. Rev. E 54, 2963 (1996).

[6] Y. D. Chong, L. Ge, H. Cao, and A. D. Stone, Coherent Perfect Absorbers: Time-Reversed Lasers, Phys. Rev. Lett. 105, 053901 (2010).

[7] D. G. Baranov, A. Krasnok, T. Shegai, A. Alú, and Y. D. Chong, Coherent perfect absorbers: Linear control of light with light, Nat. Rev. Mater. 2, 17064 (2017).

[8] A. Mostafazadeh, Spectral Singularities of Complex Scattering Potentials and Infinite Reflection and Transmission Coefficients at Real Energies, Phys. Rev. Lett. 102, 220402 (2009).

[9] B. Vainberg, On the analytical properties of the resolvent for a certain class of operator-pencils, Math. USSR Sbornik 6, 241 (1968).

[10] G. S. Guseinov, On the concept of spectral singularities, Pramana J. Phys. 73, 587 (2009).

[11] V. V. Konotop, E. Lakshtanov, and B. Vainberg, Designing lasing and perfectly absorbing potentials, Phys. Rev. A 99043838 (2019).

[12] T. Kato, Perturbation Theory of Linear Operators (Berlin: Springer, 1966).

[13] W. D. Heiss, The physics of exceptional points, J. Phys. A: Math. Theor. 45, 444016 (2012).
[14] A. A. Zharov and A. K. Kotov, Fiz. Plazmy 10, 615 (1984) (in Russian).

[15] A. Mostafazadeh, Nonlinear Spectral Singularities for Confined Nonlinearities, Phys. Rev. Lett. 110, 260402 (2013).

[16] H. Ghaemi-Dizicheh, A. Mostafazadeh, and M. Sarısaman, Nonlinear spectral singularities and laser output intensity, J. Opt. 19, 105601 (2017).

[17] K. N. Reddy and S. Dutta Gupta, Light-controlled perfect absorption of light, Opt. Lett. 38, 5252 (2013).

[18] Y. Li and C. Argypopoulos, Tunable nonlinear coherent perfect absorption with epsilon-near-zero plasmonic waveguides, Opt. Lett. 43, 8 (2018).

[19] D. A. Zezyulin and V. V. Konotop, Nonlinear currents in a ring-shaped waveguide with balanced gain and dissipation, Phys. Rev. A 94, 043853 (2016).

[20] A. Müllers, B. Santra, C. Baals, J. Jiang, J. Benary, R. Labouvie, D. A. Zezyulin, V. V. Konotop, and H. Ott, Coherent perfect absorption of nonlinear matter waves, Sci. Adv. 4, eaat6539 (2018).

[21] D. A. Zezyulin, H. Ott, and V. V. Konotop, Coherent perfect absorber and laser for nonlinear waves in optical waveguide arrays, Opt. Lett. 43, 5901 (2018).

[22] P. Devillard and B. Souillard, Polynomially decaying transmission for the nonlinear Schrödinger equation in a random medium, J. Stat. Phys. 43, 423 (1986).

[23] D. A. Zezyulin and V. V. Konotop, A universal form of localized complex potentials with spectral singularities, New J. Phys. 22, 013057 (2020).

[24] D. A. Zezyulin and V. V. Konotop, Asymmetric Perfect Absorption and Lasing of Nonlinear Waves by a Complex $\delta$-Potential, Symmetry 12, 1675 (2020). 
[25] D. A. Zezyulin, V. V. Konotop, G. Barontini, and H. Ott, Macroscopic Zeno Effect and Stationary Flows in Nonlinear Waveguides with Localized Dissipation, Phys. Rev. Lett. 109, 020405 (2012).

[26] V. S. Shchesnovich and V. V. Konotop, Control of a BoseEinstein condensate by dissipation: Nonlinear Zeno effect, Phys. Rev. A 81, 053611 (2010).

[27] F. Kh. Abdullaev, V. V. Konotop, and V. S. Shchesnovich, Linear and nonlinear Zeno effects in an optical coupler, Phys. Rev. A 83, 043811 (2011).

[28] B. Misra and E. C. G. Sudarshan, The Zeno's paradox in quantum theory, J. Math. Phys. 18, 756 (1977).

[29] W. M. Itano, D. J. Heinzen, J. J. Bollinger, and D. J. Wineland, Quantum Zeno effect, Phys. Rev. A 41, 2295 (1990).

[30] P. Facchi and S. Pascazio, Quantum Zeno dynamics: mathematical and physical aspects, J. Phys. A 41, 493001 (2008).

[31] L. P. Pitaevskii and S. Stringari, Bose-Einstein Condensation (Oxford, New York, 2003).
[32] Yu. Kivshar and G. Agrawal, Optical Solitons: From Fibers to Photonic Crystals (Academic Press, San Diego 2003).

[33] A. Mostafazadeh, Resonance phenomenon related to spectral singularities, complex barrier potential, and resonating waveguides, Phys. Rev. A 80, 032711 (2009).

[34] A. Mostafazadeh, Optical spectral singularities as threshold resonances, Phys. Rev. A 83, 045801 (2011).

[35] A. Mostafazadeh and M. Sarisaman, Lasing-threshold condition for oblique TE and TM modes, spectral singularities, and coherent perfect absorption, Phys. Rev. A 91, 043804 (2015).

[36] J. Yang, Nonlinear waves in integrable and nonintegrable systems (SIAM, Philadelphia, 2010).

[37] Handbook of Mathematical Functions, edited by M. Abramowitz and I. A. Stegun (National Bureau of Standards, Washington, DC, 1972). 